\title{
The relationship of autistic traits to taste and olfactory processing in anorexia nervosa
}

\author{
Emma Kinnaird ${ }^{1}$, Catherine Stewart ${ }^{2}$ and Kate Tchanturia ${ }^{1,3,4^{*}}$
}

\begin{abstract}
Background: There is a heightened prevalence of autism in anorexia nervosa (AN) compared to the general population. Autistic people with AN experience a longer illness duration and poorer treatment outcomes. Whether sensory differences in autism could contribute to altered taste and smell as a potential maintaining factor in AN is under-explored. The aim of this study was to explore whether autistic traits are associated with taste and olfaction differences in AN.
\end{abstract}

Methods: The study recruited $n=40$ people with AN, and $n=40$ healthy controls (HC). Smell sensitivity was measured using the Sniffin' Sticks test. Taste sensitivity was measured using taste strips. Participants self-rated their autistic traits using the Autism Spectrum Quotient.

Results: There were no significant differences on taste and olfactory outcomes between people with AN and HC. These findings did not change after controlling for the heightened levels of autistic traits in the AN group. No relationship between taste and smell outcomes and autistic traits were identified within the AN group.

Limitations: The current study is not able to draw conclusions about taste and smell processing in co-occurring autism and AN as it only measured levels of autistic traits, rather than comparing people with and without an autism diagnosis.

Conclusions: No significant associations between autistic traits and taste and smell processing in AN were identified. Future research should consider further exploring this area, including by comparing autistic women to women with AN.

Keywords: Anorexia nervosa, Eating disorders, Autism, Sensory, Taste, Olfaction

\section{Background}

Anorexia nervosa (AN) is an eating disorder (ED) characterised by the core symptoms of persistent food restriction, associated low body weight, resistance to weight gain, and body image disturbances [1]. Autistic traits are known to be heightened in AN, with around $20-25 \%$ of

* Correspondence: kate.tchanturia@kcl.ac.uk

'Department of Psychological Medicine, Institute of Psychology, Psychiatry and Neuroscience, King's College London, London, UK

${ }^{3}$ Eating Disorders Service, South London and Maudsley NHS Foundation Trust, London, UK

Full list of author information is available at the end of the article individuals presenting with clinically significant levels of autistic characteristics on clinical measures $[2,3]$. When these measures of current autistic traits are combined with developmental assessments, around 10\% of people with AN meet the diagnostic criteria for autism [4]. Significantly, autistic people with AN may experience a longer illness duration, and poorer treatment outcomes in the absence of appropriate adaptations [5-7].

Consequently, there is an increasing interest in exploring characteristics associated with autism that may play a role in maintenance models of AN, contributing to 
these poorer outcomes. For example, previous studies have investigated similarities in cognitive rigidity and theory of mind difficulties in AN and autism, both of which are thought to act as potential perpetuating factors in the illness [8-10]. To date, there is less research on the implications of sensory sensitivity in autism for AN. Sensory differences driven by alterations in bottomup processing are common in autism, to the extent that atypical responses to sensory stimuli have been included as a diagnostic criteria in the most recent edition of the Diagnostic and Statistical Manual of Mental Disorders (DSM-5) [1, 11]. Qualitative research on co-occurring autism and AN has highlighted sensory sensitivities as potentially contributing to eating difficulties in AN; however, this area remains under-explored using experimental methods $[12,13]$. This is significant as sensory sensitivity may play a role in neurobiological models of AN maintenance. Brain imaging research suggests that food restriction in this condition may be maintained by alterations in neural networks informing appetite regulation, including in areas related to taste and smell processing $[14,15]$. Specifically, a number of these studies have detected alterations in the anterior insula in response to taste stimuli [16-18]. The anterior insula is crucial to flavour perception as it integrates taste and olfactory peripheral inputs, although to date no brain imaging studies in AN have examined this area in response to olfactory stimuli [19].

Therefore, alterations in taste and smell sensitivity could potentially contribute to the dysregulation of this neural network, contributing to the key AN symptom of food restriction. Significantly, both taste and olfaction are thought to be altered in autism. Autistic people have a lowered ability to identify tastes, but appear to have normal detection thresholds [20-22]. Systematic reviews suggest that research on olfaction is more mixed [23, 24]. A recent meta-analysis on odour identification and detection thresholds found large heterogeneity in autism, with evidence for both lowered and heightened sensitivity [25].

Whether taste and olfaction are altered in AN remains unclear. As well as responding to the taste and olfactory qualities of food, the anterior insula also appears to be involved in reward and affective assessments [26, 27]. Therefore, from brain imaging evidence only it is difficult to isolate whether alterations in this area suggest altered bottom-up processing of sensory stimuli in AN, or reflect alterations in top-down processing in hedonic and reward related regions [28-30]. Recent systematic reviews on taste and smell in AN highlighted that findings in this area are mixed and inconsistent, ranging from heightened sensitivity, lowered sensitivity, or no significant findings $[31,32]$. In the context of these mixed findings, it is possible that taste and smell sensitivity are not consistently altered in AN. Rather, altered sensitivity may be related to specific states associated with AN, rather than representing a core trait of the illness itself. For example, comorbid diagnoses of mood disorders or anxiety are common in this population, and both depression and anxiety are known to be associated with altered taste and olfaction [33-36].

However, to date the potential significance of cooccurring autism in taste and smell sensitivity in AN remains under-explored. To date, two studies on smell sensitivity only have included measures of autistic traits in their analyses with conflicting results; in Bentz et al. (2017), including measures of social and communication differences associated with autism as a covariate in the analysis did not alter their findings of heightend smell sensitivity, and there was no relationship between these differences and sensory outcomes [37]. By contrast, Tonacci et al. (2019) found that adolescents with AN did not differ from healthy controls (HC) on smell sensitivity, but did find a correlation between smell sensitivity and parent-reported autistic traits [38].

Therefore, the aims of this study were to explore whether people with AN experience taste and smell differences compared to $\mathrm{HC}$, and whether taste and smell in $\mathrm{AN}$ is associated with autistic traits. In the context of previous mixed findings in the area of sensory sensitivity in AN, this study did not generate specific hypotheses prior to data collection.

\section{Methods}

\section{Participants}

In total, 40 people with $\mathrm{AN}$ and $40 \mathrm{HC}$ were recruited into the study. All participants were aged 18-55. Exclusion criteria for all participants included any current medical condition that might affect their taste or smell capacity (for example, neurological disorders such as epilepsy or Parkinson's disease). Participants experiencing a short-term condition (such as a cold or hay fever) had their testing delayed until after they had recovered.

Participants with AN were recruited from South London and Maudsley NHS Foundation Trust (SLAM) ED service. Additional participants were recruited by advertising online with a UK-based ED charity. Inclusion criteria for people with AN were a diagnosis of AN, confirmed using the Structured Clinical Interview for DSM (SCID-5) [39]. The SCID-5 was additionally used to evaluate whether the participant was experiencing restrictive AN (AN-R) or binge/purge AN (AN-BP).

HCs were included through online advertisements, and through the local university. HCs were excluded if they self-reported a diagnosis of autism, or had ever experienced an ED or other mental health condition. The absence of a previous ED or psychiatric disorder was confirmed using the SCID-5 screening tool prior to testing. The absence of a potential autism spectrum 
condition was confirmed using the Autism Quotient, with $\mathrm{HC}$ only included if they scored below the recommended 32 score threshold [40].

\section{Measures}

\section{Demographic and clinical information}

Participants were asked to self-report information on their age, ethnicity, psychiatric medication use, and whether they currently or previously smoked. People with AN self-reported their illness duration. Individuals with AN currently in treatment had their body mass index (BMI) taken from their most recent measurements in clinical notes. $\mathrm{HC}$ and individuals with $\mathrm{AN}$ not in treatment had their height and weight assessed on the day of testing.

\section{Smell sensitivity}

The Sniffin' Sticks extended test (purchased from MediSense) was used to measure three domains of smell sensitivity: odour threshold, odour discrimination, and odour identification [41]. This test was chosen as it is a standardised measure that has been used in the majority of previous research on smell sensitivity in AN [31]. The recommended procedure of the test was followed, with the exception that participants were asked to close their eyes rather than using a blindfold. During the testing, participants are asked to smell felt-tip pens filled with odours, which are held $2 \mathrm{~cm}$ in front of the centre of both nostrils. Higher scores on each test indicate higher smell sensitivity.

\section{Odour threshold}

The threshold test assesses at what threshold participants can detect the smell of $n$-butanol across 16 concentrations. Participants close their eyes, and are then presented with three pens in a forced choice paradigm: one pen contains $n$-butanol, whilst the other two contain a non-smelling solvent. Participants are instructed to identify the pen containing the odour. If the participant identifies the pen correctly twice in a row, they are presented with a set of pens at a lower concentration. If they do not correctly identify the $n$-butanol pen, the test proceeds to a set of pens at a higher concentration. The final score is the mean of the last four turning points (where the participant identifies a pen set correctly after previous incorrect identifications, or where the participant incorrectly identifies a pen set after previous correct identifications).

\section{Odour discrimination}

The discrimination test measures the ability to tell the difference between smells. Participants are asked to identify the unique odour pen from a set of 3 pens: each set has 2 pens that smell the same, and one unique pen.
This is a forced choice test, and is repeated 16 times with different smell variations. Participants closed their eyes during the test.

\section{Odour identification}

The identification test is a multiple-choice test; the researcher presents participants with one odour pen at a time. Participants then identify the smell from a card with four different options. This is repeated 16 times, with 16 different odours.

\section{Taste strips}

Taste sensitivity was assessed using taste strips, purchased from MediSense [42]. This test was used as it represents a standardised measure of a method widely used in taste research in AN, whereby taste sensitivity is assessed via the administration of tastes at varying concentrations [32]. Taste strips measure taste identification only. Participants are presented with 16 strips of filter paper, each impregnated with four ascending concentrations of the four basic tastes: sweet, salty, sour and bitter. Participants are asked to place the strip in the centre of their tongue, and to identify whether the strip was sweet, salty, sour, bitter or had no taste. Following each strip, participants rinse their mouth with water. Each correct answer yielded one point, giving a maximum score of 16 , and 4 for each individual taste quality. Scores were then summed to give a total score, and scores for each taste quality. Higher scores indicate taste smell sensitivity; lower scores indicate lower taste sensitivity.

\section{Sensory perception quotient}

Self-perceived sensitivity to external sensations, such as taste and smell, was measured using the Sensory Perception Quotient (SPQ) [43]. The SPQ was designed to measure sensory perception difficulties associated with autism in adults, and evaluates the five basic exteroceptive sensory modalities (vision, hearing, touch, smell and taste). The participant is presented with 92 different statements on sensory sensitivity, and responds on a four-point Likert scale from 'strongly agree' to 'strongly disagree'. A total of 16 items measure taste, and 16 items measure smell. In contrast to the experimental measures of taste and smell, a higher score indicates lower sensitivity, and a lower score indicates higher sensitivity. This is the first use of the SPQ in people with AN.

\section{Autism quotient}

Participants completed the adult Autism Spectrum Quotient (AQ), a self-report measure designed to screen adults for the presence of autistic traits [40]. Participants respond to 50 statements reflecting autistic traits on a four-point Likert scale from 'definitely agree' to 'definitely disagree'. A higher score indicates higher levels of 
autistic traits, with a score of $\geq 32$ indicating potentially clinically significant levels of autistic traits. The AQ was used in this current study to screen HC for clinically significant levels of autistic traits as it has shown to have good validity distinguishing cases from controls. However, recent studies suggest it may be less effective in predicting an autism diagnosis in clinical populations with high levels of suspected autistic traits [44-46]. In light of this research, the AQ was not used to categorise people with AN in this study as above or below threshold on the $\mathrm{AQ}$, but rather was used as a continuous measure of autistic traits. The AQ has been previously used in AN populations, with people with AN typically scoring higher compared to $\mathrm{HC}$ [47].

\section{Eating disorder examination questionnaire}

ED symptoms were measured using the Eating Disorder Examination questionnaire (EDE-Q) [48]. The EDE-Q is a standardised and well validated self-report measure of the severity of the characteristic psychopathology of ED. It contains 36 items which ask respondents to rate how often they have engaged in certain eating disordered behaviours or held disordered concerns over the past 28 days. The scores result in a 'global' score that represents the mean of the four subscale scores: 'eating concern', 'weight concern', 'shape concern' and 'restriction'. Higher scores indicate higher levels of ED symptoms.

\section{Hospital anxiety and depression scale}

The presence of anxiety or depression was assessed using the Hospital Anxiety and Depression Scale (HADS) [49]. The HADS is a widely used 14-item self-rating instrument for anxiety and depression in patients with both physical and mental health problems. The maximum possible score on either subscale (anxiety/depression) is 21, with higher scores indicating higher symptom levels.

\section{Procedure}

All testing took place during one session. Following informed consent, demographic and clinical information was collected from participants. Participants then completed measures in the following order: the self-report questionnaires, the smell tests and finally the taste tests. Participants were given the option to complete the selfreport questionnaires before or after the session if preferable. One participant with AN declined to complete the taste test, but participated in the smell test. A small number of participants completed the taste and smell tests, but did not fully complete all self-report questionnaires. Their data has been included in the analysis. Where group sizes vary across measures, this has been highlighted in the result tables.

\section{Statistical analysis}

Data distribution was assessed using Shapiro-Wilk tests and visual checking of histograms. The following variables were found to have a non-normal distribution and so were transformed: age, odour discrimination, and odour identification. However, the following variables could not be transformed and so were analysed using non-parametric tests: BMI, EDE-Q global scores, HADS depression scores, the vision domain of the SPQ and all taste outcomes excepting sour.

Group differences on each variable were initially explored using $t$ tests, with Mann-Whitney $U$ tests used for nonnormal distributions. Effect sizes were calculated using Cohen's $d$. Categorical variables were compared using Fisher's exact test. As the analysis involved multiple comparisons, the study used the Bonferroni correction to calculate a more conservative significance value of $p=0.005$.

Analyses of taste and smell outcomes were then repeated to control for potential confounding variables. Firstly, comparisons were rerun to exclude people who had ever smoked from each group (removing $n=10$ $\mathrm{HC}$, and $n=10$ people with AN). Secondly, to control for the potential role of medication use on sensory outcomes, the AN group was split into two sub-groups: (1) those taking psychiatric medication $(n=25)$ and (2) those not taking psychiatric medication $(n=15)$. These sub-groups were then compared on each measure.

After checking for model assumptions, ANCOVAs were performed on the smell outcomes to control for the independent contributions of anxiety and autistic traits in comparisons of sensory variables. An ANCOVA could not be performed on taste outcomes or to control for depression due to data not meeting model assumptions. To explore relationships between sensory outcomes and autistic traits, a multiple regression analysis was run within the AN group only to explore the role of autistic traits whilst controlling for the relative contributions of anxiety and depression. Finally, a Spearman's rank correlation analysis was performed to assess whether there was any association between age and sensory outcomes.

\section{Results}

\section{Participant characteristics}

Both groups were matched on age, gender, and whether they had ever smoked. Within the AN group, mean illness duration was 9.76 years $(S D=8.00)$. Thirty-four participants (85.00\%) reported experiencing AN-R, compared to 6 participants $(15.00 \%)$ with AN-BP. Eight $(20.00 \%)$ of participants were in inpatient treatment at the time of the study, $23(57.50 \%)$ participants were attending outpatient treatment, and $9(22.50 \%)$ participants were not in treatment. Twenty-six participants with AN (65.00\%) self-reported a diagnosis of at least one comorbid psychiatric condition, including anxiety, 
depression, obsessive compulsive disorder or borderline personality disorder.

Demographic and clinical characteristics for each group are summarised in Table 1. People with AN had significantly lower BMIs compared to the $\mathrm{HC}$ group, and significantly higher levels of ED symptomatology, autistic traits, anxiety and depression.

\section{Self-rated sensory sensitivity (SPQ)}

$N=38 \mathrm{HC}$, and $n=40$ people with AN, completed the SPQ (Fig. 1). People with AN scored significantly lower on touch sensitivity $(t(76)=2.90, p=0.005, d$ $=0.66)$. Lower scores on the SPQ indicate higher self-rated sensitivity, suggesting that people with AN considered themselves to be significantly more sensitive to touch compared to $\mathrm{HC}$. There were no significant differences between $\mathrm{AN}$ and $\mathrm{HC}$ groups in any other domains.

\section{Smell and taste sensitivity}

Group differences on objective measures of smell and taste sensitivity are summarised in Table 2 . There were no significant differences between groups on any outcome measure.

Excluding participants from both groups who ever smoked from the analysis did not change the direction of the results. There were no significant differences on any outcome between participants with AN not taking psychiatric medication, and those currently taking psychiatric medication. Adjusting group comparisons for the heightened levels of anxiety and autistic traits in the AN group using separate ANCOVAs did not alter the results. Result tables for these secondary analyses are located in Additional file 1.

\section{Relationships between clinical variables}

Regression analyses were performed within the AN group to explore the relative contributions of anxiety, depression and autistic traits to sensory measures. With the significance level set at $p=0.005$, these variables were not found to individually contribute to any sensory outcome at a significant level (Table 3).

A correlation analysis was also performed to explore whether there was an association between age and sensory outcomes. No significant correlations were found (Table 4).

\section{Discussion}

The aims of this study were to explore whether people with AN experience taste and smell differences compared to $\mathrm{HC}$, and whether taste and smell in AN is associated with autistic traits. The analyses identified no significant differences on taste and smell outcomes between people with $\mathrm{AN}$ and $\mathrm{HC}$, and no significant associations between taste, smell and autistic traits within the AN group.

Overall, people with AN did not exhibit significant differences on objective measures of sensory sensitivity compared to $\mathrm{HC}$, including after controlling for the potential role of autistic traits. If the absence of significant differences identified in the analysis reflects a true absence of difference, this could suggest that identified alterations in appetite neurocircuitry in AN are driven by differences in top-down processing relating to perceived reward, hedonic and affective salience, rather than bottom-up differences at a sensory level [16-18]. By contrast, previous research in autism has found lowered taste sensitivity [20,21]. Findings on smell sensitivity in autism are comparably less consistent, with evidence for both heightened and lowered olfaction [25, 50]. If AN and autism are associated with different processes relating to taste, and potentially smell, this could explain why autistic traits were not found to be related to sensory sensitivity in AN in our study.

However, the current study cannot draw conclusions surrounding whether $\mathrm{AN}$ and autism are associated with

Table 1 Group demographic and clinical characteristics

\begin{tabular}{|c|c|c|c|c|c|}
\hline & HC mean $(S D)(n=40)$ & AN mean $(S D)(n=40)$ & Test statistic & $p$ & Effect size $(d(95 \% C())$ \\
\hline Age (years) & $26.45(7.55)$ & $26.65(8.60)$ & $t(78)=-0.33$ & 0.741 & $-0.07(-0.51-0.36)$ \\
\hline Gender & $\begin{array}{l}n=38 \text { female }(95.00 \%) \\
n=2 \text { male }(5.00 \%)\end{array}$ & $\begin{array}{l}n=38 \text { female }(95.00 \%) \\
n=2 \text { male }(5.00 \%)\end{array}$ & & 1.00 & \\
\hline BMI* & $22.5(4.3)$ & $15.75(1.22)$ & $U=0$ & $\leq 0.001$ & $2.92(2.27-3.55)$ \\
\hline Smoking (\% never smoked) & $n=30(75.00 \%)$ & $n=30(75.00 \%)$ & & 1.00 & \\
\hline Psychiatric medication (\% currently taking) & $n=0(0.00 \%)$ & $n=25(62.50 \%)$ & & $\leq 0.001$ & \\
\hline EDE-Q global* & $0.58(0.9) n=39$ & $4.05(1.79) n=39$ & $U=74$ & $\leq 0.001$ & $-2.90(-3.54-2.26)$ \\
\hline AQ & $12.48(6.74)$ & $23.55(10.26)$ & $t(78)=-5.71$ & $\leq 0.001$ & $-1.28(-1.75-0.79)$ \\
\hline HADS depression* & $2(4) n=39$ & $9(5) n=39)$ & $U=174.5$ & $\leq 0.001$ & $-1.79(-2.32-1.26)$ \\
\hline HADS anxiety & $6.08(3.80) n=39$ & $13.00(4.63) n=39$ & $t(76)=-7.21$ & $\leq 0.001$ & $-1.63(-2.14-1.12)$ \\
\hline
\end{tabular}

*Indicates non-normally distributed data. Medians and interquartile ranges presented instead of means/standard deviations (SD). Effect sizes presented with $95 \%$ confidence intervals $(C l)$ 


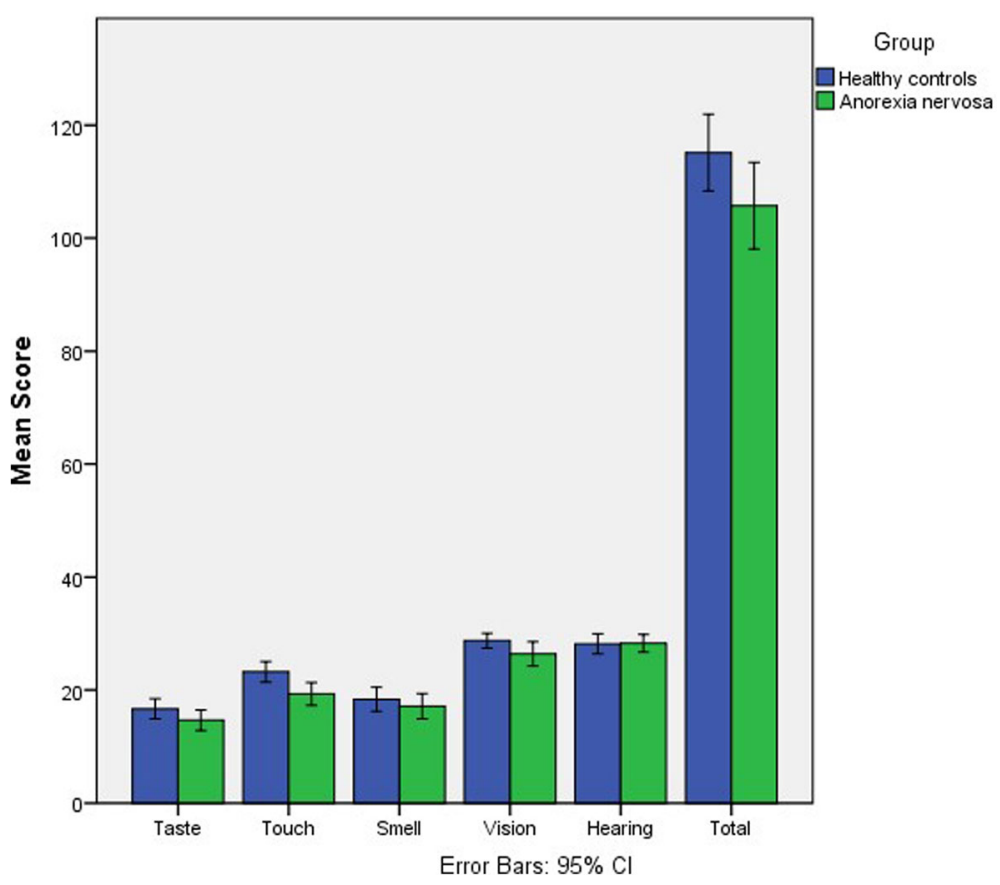

Fig. 1 Group scores on the SPQ, summarised by sensory domain and total score

different sensory processing as an autism comparison group was not included. Moreover, previous research on smell and taste processing in autism may lack comparability to studies in AN, including the present paper. The current study, and most previous research on taste and smell sensitivity in AN, has used a predominantly female adult sample [31, 32]. By contrast, research in this area in autism has used predominantly male samples, with a greater focus on children [23-25, 50]. Both age and gender are known to affect sensory processing, particularly in the area of olfaction. Research suggests that olfactory performance improves throughout childhood, reaching adult levels in the later teenage period [51]. Both taste and smell performance then declines after the sixth decade of life [52, 53]. Women also appear to outperform men on smell tests, and there is some evidence that women process tastes differently in the brain $[54,55]$. In this context, it is difficult to draw firm conclusions comparing research from these different fields due to the difference in samples. Future research should consider comparing taste and smell processing in autistic people to people with $\mathrm{AN}$, with age and gender-matched samples, to further explore this area.

Previous research on whether there is a relationship between smell sensitivity and autistic traits in AN has yielded mixed results (to date, no study has examined taste sensitivity). One study found a relationship between parent-reported autistic traits and olfaction [38],

Table 2 Taste and smell group outcomes

\begin{tabular}{llllll}
\hline & HC mean $(S D)(n=40)$ & AN mean $(S D)(n=40)$ & Test statistic & $p$ & Effect size $(d(95 \%$ C $))$ \\
\hline Smell total & $36.28(4.07)$ & $35.7(4.36)$ & $t(78)=0.62$ & 0.539 & $0.14(0.30-0.58)$ \\
$\quad$ Odour threshold & $9.88(3.09)$ & $10.80(2.84)$ & $t(78)=-1.38$ & 0.170 & $-0.31(-0.75-0.13)$ \\
Odour discrimination & $12.98(1.69)$ & $12.15(2.35)$ & $t(78)=1.72$ & 0.089 & $0.39(-0.06-0.83)$ \\
Odour identification & $13.43(1.85)$ & $12.75(1.69)$ & $t(78)=1.99$ & 0.050 & $0.45(0.00-0.89)$ \\
& HC mean (SD) $(n=40)$ & AN mean (SD) $(n=39)$ & & & \\
Taste total* & $13(4)$ & $11(5)$ & $U=628.5$ & 0.135 & $0.36(-0.09-0.80)$ \\
Sweet* & $4(1)$ & $4(1)$ & $U=719$ & 0.502 & $0.17(-0.27-0.61)$ \\
Sour & $2.40(1.08)$ & $1.82(1.30)$ & $t(77)=2.16$ & 0.034 & $0.49(0.04-0.93)$. \\
Salty* & $4(1)$ & $4(1)$ & $U=742.5$ & 0.686 & $0.11(-0.34-0.55)$ \\
Bitter* & $3(2)$ & $3(2)$ & $U=692.5$ & 0.369 & $0.21(-0.23-0.65)$ \\
\hline
\end{tabular}

*Indicates non-normally distributed data. Medians and interquartile ranges presented instead of means/standard deviations (SD) 
Table 3 Regression analysis of relative contribution of anxiety, depression and autistic traits to sensory outcomes

\begin{tabular}{|c|c|c|c|c|c|c|c|c|c|}
\hline & \multicolumn{3}{|c|}{ Anxiety (HADS) } & \multicolumn{3}{|c|}{ Depression (HADS) } & \multicolumn{3}{|c|}{ Autistic traits (AQ) } \\
\hline & $B$ & $t$ & $p$ & $B$ & $t$ & $p$ & $B$ & $t$ & $p$ \\
\hline Smell total & 0.24 & 1.35 & 0.186 & -0.07 & -0.39 & 0.702 & -0.02 & -0.11 & 0.913 \\
\hline Odour threshold & 0.01 & 0.03 & 0.975 & 0.16 & 0.83 & 0.414 & 0.05 & 0.29 & 0.772 \\
\hline Odour discrimination & 0.37 & 2.13 & 0.040 & -0.06 & -0.34 & 0.738 & -0.15 & -0.84 & 0.407 \\
\hline Odour identification & 0.10 & 0.57 & 0.570 & -0.38 & -2.10 & 0.043 & 0.07 & 0.38 & 0.706 \\
\hline Taste total & 0.15 & 0.83 & 0.411 & -0.19 & -0.99 & 0.329 & -0.02 & -0.12 & 0.906 \\
\hline Sweet & 0.07 & 0.40 & 0.691 & -0.19 & -0.98 & 0.333 & 0.02 & 0.10 & 0.925 \\
\hline Sour & 0.32 & 1.83 & 0.076 & -0.27 & -1.49 & 0.145 & 0.06 & 0.35 & 0.726 \\
\hline Salty & 0.17 & 0.94 & 0.354 & -0.14 & -0.76 & 0.452 & -0.05 & -0.26 & 0.795 \\
\hline Bitter & -0.11 & -0.58 & 0.563 & 0.04 & 0.20 & 0.844 & -0.08 & -0.43 & 0.669 \\
\hline
\end{tabular}

and another found no relationship between social and communicative characteristics of autism and smell outcomes [37]. It should be noted that autistic traits have not consistently found to be related to smell sensitivity even in autistic populations, with studies finding conflicting results [50, 56, 57]. One possibility for these mixed findings is that the heightened levels of autistic traits influence smell outcomes via a secondary, mediating variable. For example, anxiety and sensory sensitivity are known to be interrelated features in autism [58]. A strength of the present study is that the relationship of autistic traits and sensory outcomes in AN was analysed relative to the contributions of anxiety and depression using a multiple regression analysis. This is important as both anxiety and depression are known to influence taste and smell processing, including in AN [33, 34, 37]. The lifetime prevalence rate of anxiety in autistic adults is $42 \%$, and $37 \%$ for depression [59]. Additionally, anxiety has been shown to correlate with autistic traits in AN [60]. Therefore, the finding of this study that autistic traits were not significantly related to objective measures of taste and smell sensitivity are likely to be more robust against the influence of these interrelated variables.

Table 4 Correlation analysis between age and sensory outcomes

\begin{tabular}{lll}
\hline & Age (years) \\
\cline { 2 - 3 } & $r$ & $p$ \\
\hline Smell total & 0.22 & 0.171 \\
Odour threshold & 0.06 & 0.701 \\
Odour discrimination & 0.024 & 0.135 \\
Odour identification & 0.34 & 0.031 \\
Taste total & 0.13 & 0.419 \\
Sweet & -0.08 & 0.599 \\
Sour & 0.11 & 0.515 \\
Salty & -0.02 & 0.911 \\
Bitter & 0.18 & 0.287 \\
\hline
\end{tabular}

However, the current study did not explore IQ as a potential confounding variable, although it has previously been identified as a moderating factor in smell sensitivity in autism [25]. Additionally, this study did not control for age beyond excluding people aged over 55 years, although no significant relationship between age and sensory outcomes was identified in the current study. Therefore, future research exploring smell sensitivity, autism and anorexia could further consider the potential role of mediating variables in this relationship.

On self-report measures people with AN exhibited no significant differences in taste and smell sensitivity compared to HC. Previous research indicates that people with AN may rate themselves as significantly more sensitive to sensations, and may be more likely to attempt to avoid sensory stimuli compared to $\mathrm{HC}[61,62]$. As the self-report measures in this study reflect the objective outcomes, it is possible that the SPQ represents an accurate measure of sensory sensitivity only in this population, whereas previous studies additionally measured affective or hedonic responses to sensory stimuli $[61,62]$. In the current study, people with AN only rated themselves as more sensitive to touch. The area of touch sensitivity has primarily been explored through the paradigm of affective touch: people with AN perceive affective, interpersonal touching as less pleasurable compared to HC [63]. Future research could consider if the pleasurable aspects of interpersonal touch may also be related to an underlying sensory hypersensitivity to touch itself.

\section{Limitations}

A limitation of this study was that it measured self-rated levels of autistic traits in people with $\mathrm{AN}$, rather than comparing autistic people with AN to those with AN only. Therefore, it cannot draw conclusions on whether these two groups do experience differences in taste and smell sensitivity. Further research should consider autistic people to people with AN only, including matching 
for age and gender. Future research could also explore using clinical and developmental autism assessments to establish a co-occurring autism/AN group, and compare to people with AN only and HC. An additional limitation of this study is that whilst it used a heterogenous AN sample that may better reflect clinical variety, the sample was not large enough to control within the analysis for potential confounding factors introduced by this heterogeneity. For example, the current study did not explore the potential role of illness duration, BMI or comorbidities beyond anxiety and depression.

Furthermore, although the findings of this study were non-significant, the methods used cannot account for whether this reflects a true absence of difference between groups, or whether these non-significant findings result from chance or a lack of power [64]. However, sample size calculations to determine the minimum sample size required for adequate study power were calculated a priori to recruitment on the basis of two previous studies in AN which measured the same taste and smell outcomes as the present research, and which reported means and standard deviations $[65,66]$. These calculations suggested that a minimum sample size approximating $n=20$ in each group would give sufficient statistical power, although the current study recruited larger groups due to its aim to investigate potential covariates. This indicates that the lack of significant findings is unlikely to be due to a lack of power. Additionally, the finding that confidence intervals for the majority of taste and smell outcomes includes 0 supports an absence of significant difference. However, without a Bayesian approach to analysis the current study cannot precisely quantify the level of evidence for whether there is truly no difference between groups.

\section{Conclusions}

The study found that taste and smell sensitivity was not significantly altered in AN, with no significant relationship between these outcomes and autistic traits within the AN group. In the context of previous studies suggesting altered taste and smell sensitivity in autism, future research should explore sensory differences in autistic people compared to people with AN, and implications for individuals with co-occurring autism and AN.

\section{Supplementary information}

Supplementary information accompanies this paper at https://doi.org/10. 1186/s13229-020-00331-8.

Additional file 1. Secondary Analysis Results. File including results tables for secondary analyses.

\section{Abbreviations}

AN: Anorexia nervosa; AN-BP: Anorexia nervosa, binge-purge subtype; ANR: Anorexia nervosa, restrictive subtype; AQ: Autism Quotient (Adult);
BMI: Body mass index; Cl: Confidence intervals; DSM: Diagnostic and Statistical Manual of Mental Disorders; ED: Eating disorder; EDE-Q: Eating Disorder Examination Questionnaire; HADS: Hospital Anxiety and Depression Scale; HC: Healthy control; SCID-5: Structured Clinical Interview for DSM;

SD: Standard deviations; SLAM: South London and Maudsley NHS

Foundation Trust; SPQ: Sensory Perception Quotient; UK: United Kingdom

\section{Acknowledgements}

The authors would like to thank the UK ED charity Beat for their support with study recruitment.

\section{Authors' contributions}

All authors contributed to the design of the study. EK collected the data and performed the analysis, with supervision from KT and CS. EK wrote the first draft of the manuscript, with subsequent input from KT and CS. All authors read and approved the final manuscript.

\section{Funding}

EK was supported by a Medical Research Council Doctoral Training Partnership studentship (MR/N013700/1). KT would like to acknowledge support from the Health foundation, an independent charity committed to bring better health care for people in the UK (AIMS ID: 1115447). KT would also like to acknowledge two grants from the MRC-MRF fund (MR/S020381/ 1. BiomaRkers for Anorexla NErvosa and autism spectrum Disorders- longitudinal study; and MR/R004595/1 The Triple A study (Adolescents with Anorexia and Autism): A search for biomarkers).

\section{Availability of data and materials}

The datasets used and/or analysed during the current study are available from the corresponding author on reasonable request.

\section{Ethics approval and consent to participate}

All participants gave written informed consent prior to participation, and the study received ethnical approval from the North East Newcastle \& North

Tyneside 2 research ethics committee (18/NE/0193).

\section{Consent for publication}

Not applicable.

\section{Competing interests}

The authors declare that they have no competing interests.

\section{Author details}

${ }^{1}$ Department of Psychological Medicine, Institute of Psychology, Psychiatry and Neuroscience, King's College London, London, UK. ' Maudsley Centre for Child and Adolescent Eating Disorders, South London and Maudsley NHS Foundation Trust, London, UK. ${ }^{3}$ Eating Disorders Service, South London and Maudsley NHS Foundation Trust, London, UK. ${ }^{4}$ Department of Psychology, Illia State University, Tbilisi, Georgia.

Received: 29 November 2019 Accepted: 27 March 2020

Published online: 10 April 2020

\section{References}

1. American Psychiatric Association. Diagnostic and statistical manual of mental disorders. 5 ed. Arlington, VA: American Psychiatric Publishing; 2013.

2. Westwood H, Mandy W, Tchanturia K. Clinical evaluation of autistic symptoms in women with anorexia nervosa. Molecular Autism. 2017;8.

3. Bentz M, Jepsen JR, Pedersen T, Bulik CM, Pedersen L, Pagsberg AK, et al. Impairment of social function in young females with recent-onset anorexia nervosa and recovered individuals. The Journal of adolescent health : official publication of the Society for Adolescent Medicine. 2017;60(1):23-32.

4. Westwood H, Mandy W, Simic M, Tchanturia K. Assessing ASD in adolescent females with anorexia nervosa using clinical and developmental measures: a preliminary investigation. J Abnormal Child Psychol. 2017.

5. Tchanturia K, Larsson E, Adamson J. How anorexia nervosa patients with high and low autistic traits respond to group cognitive remediation therapy. BMC Psychiatry. 2016;16.

6. Nielsen S, Anckarsater H, Gillberg C, Rastam M, Wentz E. Effects of autism spectrum disorders on outcome in teenage-onset anorexia nervosa 
evaluated by the Morgan-Russell outcome assessment schedule: a controlled community-based study. Molecular Autism. 2015;6.

7. Stewart CS, McEwen FS, Konstantellou A, Eisler I, Simic M. Impact of ASD Traits on treatment outcomes of eating disorders in girls. European Eating Disorders Review. 2017;25(2):123-8.

8. Westwood H, Stahl D, Mandy W, Tchanturia K. The set-shifting profiles of anorexia nervosa and autism spectrum disorder using the Wisconsin Card Sorting Test: a systematic review and meta-analysis. Psychological Medicine. 2016;46(9):1809-27.

9. Treasure J, Schmidt U. The cognitive-interpersonal maintenance model of anorexia nervosa revisited: a summary of the evidence for cognitive, socioemotional and interpersonal predisposing and perpetuating factors. Journal of Eating Disorders2013.

10. Leppanen J, Sedgewick F, Treasure J, Tchanturia K. Differences in the theory of mind profiles of patients with anorexia nervosa and individuals on the autism spectrum: a meta-analytic review. Neurosci Biobehav Rev. 2018;90: 146-63.

11. Robertson CE, Baron-Cohen S. Sensory perception in autism. Nat Rev Neurosci. 2017;18(11):671-84.

12. Kinnaird E, Norton C, Tchanturia K. Clinicians' views on working with anorexia nervosa and autism spectrum disorder comorbidity: a qualitative study. BMC Psychiatry. 2017;17:292.

13. Kinnaird E, Norton C, Stewart C, Tchanturia K. Same behaviours, different reasons: what do patients with co-occurring anorexia and autism want from treatment? Int Rev Psychiatry. 2019;31(4):308-17.

14. Kaye WH. Eating disorders: hope despite mortal risk. Am J Psychiatry. 2009; 166(12):1309-11.

15. Kaye WH, Wierenga CE, Bailer UF, Simmons AN, Bischoff-Grethe A. Nothing tastes as good as skinny feels: the neurobiology of anorexia nervosa. Trends Neurosci. 2013;36(2):110-20.

16. Oberndorfer TA, Frank GKW, Simmons AN, Wagner A, McCurdy D, Fudge JL, et al. Altered insula response to sweet taste processing after recovery from anorexia and bulimia nervosa. Am J Psychiatry. 2013;170(10):1143-51.

17. Frank GK, Shott ME, Hagman JO, Mittal VA. Alterations in brain structures related to taste reward circuitry in ill and recovered anorexia nervosa and in bulimia nervosa. Am J Psychiatry. 2013;170(10):1152-60.

18. Frank GK, Shott ME, Keffler C, Cornier M-A. Extremes of eating are associated with reduced neural taste discrimination. International Journal of Eating Disorders. 2016;49(6):603-12.

19. Small DM. Flavor is in the brain. Physiology \& behavior. 2012;107(4):540-52.

20. Tavassoli T, Baron-Cohen S. Taste identification in adults with autism spectrum conditions. Journal of Autism and Developmental Disorders. 2012; 42(7):1419-24.

21. Bennetto L, Kuschner ES, Hyman SL. Olfaction and taste processing in autism. Biological psychiatry. 2007;62(9):1015-21.

22. Damiano CR, Aloi J, Burrus C, Garbutt JC, Kampov-Polevoy AB, Dichter GS. Intact hedonic responses to sweet tastes in autism spectrum disorder. Research in autism spectrum disorders. 2014;8(3):230-6.

23. Tonacci A, Billeci L, Tartarisco G, Ruta L, Muratori F, Pioggia G, et al. Olfaction in autism spectrum disorders: a systematic review. Child Neuropsychology. 2017;23(1):1-25.

24. Boudjarane MA, Grandgeorge M, Marianowski R, Misery L, Lemonnier E. Perception of odors and tastes in autism spectrum disorders: a systematic review of assessments. Autism Research. 2017;10(6):1045-57.

25. Larsson M, Tirado C, Wiens S. A meta-analysis of odor thresholds and odor identification in autism spectrum disorders. Front Psychol. 2017;8:679.

26. Small DM, Zatorre RJ, Dagher A, Evans AC, Jones-Gotman M. Changes in brain activity related to eating chocolate: from pleasure to aversion. Brain. 2001;124(9):1720-33.

27. Craig AD. How do you feel - now? The anterior insula and human awareness. Nature Reviews Neuroscience. 2009;10(1):59-70.

28. Brooks SJ, Barker GJ, O'Daly OG, Brammer M, Williams SCR, Benedict C, et al. Restraint of appetite and reduced regional brain volumes in anorexia nervosa: a voxel-based morphometric study. Bmc Psychiatry. 2011;11.

29. Titova OE, Hjorth OC, Schiöth HB, Brooks SJ. Anorexia nervosa is linked to reduced brain structure in reward and somatosensory regions: a metaanalysis of VBM studies. BMC Psychiatry. 2013;13(1):110.

30. Keating C, Tilbrook AJ, Rossell SL, Enticott PG, Fitzgerald PB. Reward processing in anorexia nervosa. Neuropsychologia. 2012;50(5):567-75.
31. Islam MA, Fagundo AB, Arcelus J, Aguera Z, Jimenez-Murcia S, FernandezReal JM, et al. Olfaction in eating disorders and abnormal eating behavior: a systematic review. Front Psychol. 2015;6.

32. Kinnaird E, Stewart C, Tchanturia K. Taste sensitivity in anorexia nervosa: a systematic review. International Journal of Eating Disorders. 2018.

33. Lombion-Pouthier S, Vandel P, Nezelof S, Haffen E, Millot JL. Odor perception in patients with mood disorders. Journal of Affective Disorders. 2006;90(2-3):187-91.

34. Buron E, Bulbena A. Olfaction in affective and anxiety disorders: a review of the literature. Psychopathology. 2013;46(2):63-74.

35. Kaye WH, Bulik CM, Thornton L, Barbarich N, Masters K. Price Fdn Collaborative G. Comorbidity of anxiety disorders with anorexia and bulimia nervosa. Am J Psychiatry. 2004;161(12):2215-21.

36. Salbach-Andrae H, Lenz K, Simmendinger N, Klinkowski N, Lehmkuhl U, Pfeiffer E. Psychiatric comorbidities among female adolescents with anorexia nervosa. Child Psychiatry and Human Development. 2008;39(3): 261-72.

37. Bentz M, Guldberg J, Vangkilde S, Pedersen T, Plessen KJ, Jepsen JR. Heightened olfactory sensitivity in young females with recent-onset anorexia nervosa and recovered individuals. PLoS One. 2017;12(1):e0169183.

38. Tonacci A, Calderoni S, Billeci L, Maestro S, Fantozzi P, Ciuccoli F, et al. Autistic traits impact on olfactory processing in adolescent girls with anorexia nervosa restricting type. Psychiatry Res. 2019;274:20-6.

39. First M, Williams J, Karg R, Spitzer R. Structured clinical interview for DSM-5, Research Version. Arlington, VA: American Psychiatric Association; 2015.

40. Baron-Cohen S, Wheelwright S, Skinner R, Martin J, Clubley E. The autismspectrum quotient $(\mathrm{AQ})$ : evidence from Asperger syndrome/highfunctioning autism, males and females, scientists and mathematicians. Journal of Autism and Developmental Disorders. 2001;31(1):5-17.

41. Hummel T, Sekinger B, Wolf SR, Pauli E, Kobal G. 'Sniffin' Sticks': olfactory performance assessed by the combined testing of odor identification, odor discrimination and olfactory threshold. Chemical Senses. 1997;22(1):39-52.

42. Mueller C, Kallert S, Renner B, Stiassny K, Temmel AFP, Hummel T, et al. Quantitative assessment of gustatory function in a clinical context using impregnated "taste strips". Rhinology. 2003;41(1):2-6.

43. Tavassoli T, Hoekstra RA, Baron-Cohen S. The sensory perception quotient (SPQ): development and validation of a new sensory questionnaire for adults with and without autism. Molecular Autism. 2014;5(1):29.

44. Ashwood KL, Gillan N, Horder J, Hayward H, Woodhouse E, McEwen FS, et al. Predicting the diagnosis of autism in adults using the autismspectrum quotient (AQ) questionnaire. Psychological medicine. 2016;46(12): 2595-604.

45. Sizoo BB, Horwitz EH, Teunisse JP, Kan CC, Vissers C, Forceville EJM, et al Predictive validity of self-report questionnaires in the assessment of autism spectrum disorders in adults. Autism. 2015;19(7):842-9.

46. Conner CM, Cramer RD, McGonigle JJ. Examining the diagnostic validity of autism measures among adults in an outpatient clinic sample. Autism in Adulthood. 2019;1(1):60-8.

47. Westwood H, Eisler I, Mandy W, Leppanen J, Treasure J, Tchanturia K. Using the autism-spectrum quotient to measure autistic traits in anorexia nervosa: a systematic review and meta-analysis. Journal of Autism and Developmental Disorders. 2016;46(3):964-77.

48. Fairburn CG, Beglin SJ. Eating disorder examination questionnaire (EDE-Q 6 . 0). In: Fairburn CG, editor. Cognitive behaviour therapy and eating disorders. New York: Guilford Press; 2008.

49. Zigmond AS, Snaith RP. The hospital anxiety and depression scale. Acta Psychiatrica Scandinavica. 1983;67(6):361-70.

50. Muratori F, Tonacci A, Billeci L, Catalucci T, Igliozzi R, Calderoni S, et al. Olfactory processing in male children with autism: atypical odor threshold and identification. Journal of Autism and Developmental Disorders. 2017; 47(10):3243-51.

51. Cameron EL. Olfactory perception in children. World J Otorhinolaryngol Head Neck Surg. 2018;4(1):57-66.

52. Mullol J, Alobid I, Mariño-Sánchez F, Quintó L, de Haro J, Bernal-Sprekelsen $\mathrm{M}$, et al. Furthering the understanding of olfaction, prevalence of loss of smell and risk factors: a population-based survey (OLFACAT study). BMJ Open. 2012;2(6):e001256.

53. Boyce JM, Shone GR. Effects of ageing on smell and taste. Postgrad Med J. 2006;82(966):239-41. 
54. Haase L, Green E, Murphy C. Males and females show differential brain activation to taste when hungry and sated in gustatory and reward areas. Appetite. 2011;57(2):421-34.

55. Sorokowski P, Karwowski M, Misiak M, Marczak MK, Dziekan M, Hummel T, et al. Sex differences in human olfaction: a meta-analysis. Frontiers in Psychology. 2019;10(242).

56. Dudova I, Hrdlicka M. Olfactory functions are not associated with autism severity in autism spectrum disorders. Neuropsychiatric disease and treatment. 2013;9:1847-51.

57. Ashwin C, Chapman E, Howells J, Rhydderch D, Walker I, Baron-Cohen S. Enhanced olfactory sensitivity in autism spectrum conditions. Molecular Autism. 2014;5(1):53

58. Neil L, Olsson NC, Pellicano E. The relationship between intolerance of uncertainty, sensory sensitivities, and anxiety in autistic and typically developing children. Journal of Autism and Developmental Disorders. 2016; 46(6):1962-73.

59. Hollocks MJ, Lerh JW, Magiati I, Meiser-Stedman R, Brugha TS. Anxiety and depression in adults with autism spectrum disorder: a systematic review and meta-analysis. Psychological Medicine. 2018;49(4):559-72.

60. Tchanturia K, Smith E, Weineck F, Fidanboylu E, Kern N, Treasure J, et al. Exploring autistic traits in anorexia: a clinical study. Molecular Autism. 2013; 4.

61. Zucker NL, Merwin RM, Bulik CM, Moskovich A, Wildes JE, Groh J. Subjective experience of sensation in anorexia nervosa. Behav Res Ther. 2013;51(6): 256-65.

62. Brand-Gothelf A, Parush S, Eitan Y, Admoni S, Gur E, Stein D. Sensory modulation disorder symptoms in anorexia nervosa and bulimia nervosa: a pilot study. International Journal of Eating Disorders. 2016:49(1):59-68.

63. Crucianelli L, Cardi V, Treasure J, Jenkinson PM, Fotopoulou A. The perception of affective touch in anorexia nervosa. Psychiatry Res. 2016;239: 72-8.

64. Gates S, Ealing E. Reporting and interpretation of results from clinical trials that did not claim a treatment difference: survey of four general medical journals. BMJ Open. 2018;9:e024785.

65. Aschenbrenner K, Scholze N, Joraschky P, Hummel T. Gustatory and olfactory sensitivity in patients with anorexia and bulimia in the course of treatment. J. Psychiatr. Res. 2008:43(2):129-37.

66. Dazzi F, De Nitto S, Zambetti G, Loriedo C, Ciofalo A. Alterations of the olfactory-gustatory functions in patients with eating disorders. Eur. Eat. Disorders. Rev. 2013;21:382-5.

\section{Publisher's Note}

Springer Nature remains neutral with regard to jurisdictional claims in published maps and institutional affiliations.

Ready to submit your research? Choose BMC and benefit from:

- fast, convenient online submission

- thorough peer review by experienced researchers in your field

- rapid publication on acceptance

- support for research data, including large and complex data types

- gold Open Access which fosters wider collaboration and increased citations

- maximum visibility for your research: over $100 \mathrm{M}$ website views per year

At $\mathrm{BMC}$, research is always in progress.

Learn more biomedcentral.com/submissions 